\title{
Fast Computing on Vehicle Dynamics Using Chebyshev Series Expansions
}

\author{
IEEE/ASME TRANSACTIONS ON MECHATRONICS
}

\author{
REGULAR PAPER
}

\author{
COVER LETTER
}

Authors: López A., Moriano C., Olazagoitia J.L., and Páez J.

\begin{abstract}
Alberto López : Universidad Antonio de Nebrija. Industrial Engineering Department. C/Pirineos 55, 28040 - Madrid, (Spain) e-mail: alopezro@nebrija.es . Phone: 00349145211 00; Fax: 0034914521110 Corresponding Author.

Cristina Moriano: Universidad Antonio de Nebrija. Industrial Engineering Department. C/Pirineos 55, 28040 - Madrid, (Spain) e-mail: cmoriano@nebrija.es. Phone: 00349145211 00; Fax: 0034914521110

José Luis Olazagoitia: Universidad Antonio de Nebrija. Industrial Engineering Department. C/Pirineos 55, 28040 - Madrid, (Spain)

e-mail: jolazago@nebrija.es . Phone: 00349145211 00; Fax: 0034914521110

Francisco Javier Páez: Universidad Politécnica de Madrid. INSIA. Instituto de Investigación del Automóvil. Campus Sur UPM, Carretera de Valencia (A-3), km 7, 28031 Madrid, (Spain). e-mail: franciscojavier.paez@upm.es .Phone 00349133653 00; Fax 0034913365302
\end{abstract}




\title{
Fast Computing on Vehicle Dynamics Using Chebyshev Series Expansions
}

\author{
López A., Moriano C., Olazagoitia J.L., and Páez J.
}

\begin{abstract}
This article focusses on faster computation techniques to integrate mechanical models in electronic advanced active safety applications. It shows the different techniques of approximation in series of functions and differential equations applied to vehicle dynamics. This allows the achievement of approximate polynomial and rational solutions with a very fast and efficient computation. Firstly, the whole theoretical basic principles related to the techniques used are presented: orthogonality of functions, function expansion in Chebyshev and Jacobi series, approximation through rational functions, the Minimax-Remez algorithm, orthogonal rational functions (ORF's) and the perturbation of dynamic systems theory, that reduces the degree of the expansion polynomials used.

As an application, it is shown the obtaining of approximate solutions to the longitudinal dynamics, vertical dynamics, steering geometry and a tyre model, all obtained through development in series of orthogonal functions with a computation much faster than those of its equivalents in the classic vehicle theory. These polynomial partially symbolic solutions present very low errors and very favourable analytical properties due to their simplicity, becoming ideal for real time computation as those required for the simulation of evasive manoeuvres prior a crash. This set of techniques had never been applied to vehicle dynamics before.
\end{abstract}

Keywords: Fast solvers, Chebyshev series, Theory of Approximation, Vehicle Dynamics.

\section{INTRODUCTION}

WITHIN the work areas about active safety and collision avoidance, nowadays it is being developed an intense research on obstacle detection and image processing, see [1], [2],[3] and [4], as not all the problems of global environment

recognition surrounding a vehicle with its multiple configurations and situations are resolved.

But even in the case of a complete and precise recognition of the vehicle's environment, the next step would be the definition of the vehicle's behaviour in an imminent danger situation, its correct action in order to avoid an accident or reduce its

Submitted: April-3-2014

This work has been supported by the SEGVAUTO research group of the Comunidad de Madrid.

A. López, is with the Industrial Engineering Department. Universidad Antonio de Nebrija. C/Pirineos 55, 28040 - Madrid, (Spain) (e-mail: alopezro@nebrija.es).

C. Moriano is with the Industrial Engineering Department. Universidad Antonio de Nebrija. C/Pirineos 55, 28040 - Madrid, (Spain) (e-mail: cmoriano@nebrija.es). seriousness and the vehicle's response within its environment when performing that evasive action. In order to evaluate those possible evasive actions, it is necessary to process a simulation model of the vehicle working faster than real time.

Following the works by Margolis [5], López [6] and Moriano [7] the study of the dynamic behaviour of a vehicle in a precollision scenario, with at least a second in advance, requires a model with ten degrees of freedom at least, plus a tyre model. Supposing a scenario where a vehicle detects an obstacle, for instance a pedestrian in the middle of the road (see Fig. 1), there may be several possible escape trajectories, depending on whether the evasive action is braking, a turn of the steering wheel or a combination of both.

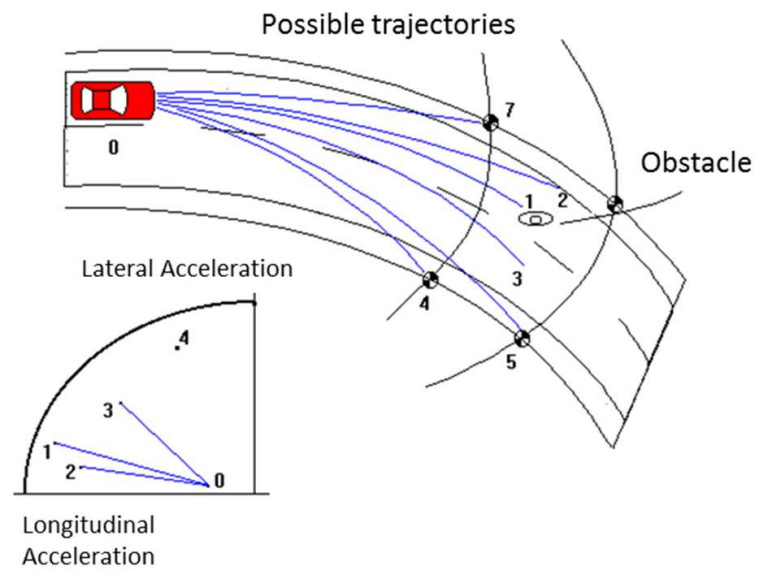

Fig. 1. Pre-collision scenario.

Evaluating all the possible trajectories and selecting the one requiring less force in the contact tyre-ground, keeping the contact stress within the friction ellipse (see Fig. 1) and a safe evasive manoeuvre, implies a very large computational load. Therefore, both the vehicle and tyre model in 10 DOF must be resolved faster than real time to evaluate trajectories and the active safety margin in the friction ellipse in the following instants through a real time predictive simulation.

J.L. Olazagoitia is with the Industrial Engineering Department. Universidad Antonio de Nebrija. C/Pirineos 55, 28040 - Madrid, (Spain) (e-mail: jolazago@nebrija.es).

J. Páez is with the INSIA Research Institute. Universidad Politécnica de Madrid. Campus Sur UPM, Carretera de Valencia (A-3), km 731 Madrid, (Spain) (email: franciscojavier.paez@upm.es). 
The real time condition demands the time the computer takes to calculate an integration step $\left(t_{c}\right)$ to be less than the actual integration step $\left(\mathrm{t}_{\mathrm{I}}\right)$. In order to guarantee the correct convergence of the equations system of the complete model of a 10 DOF vehicle plus a tyre model, the integration step, $\mathrm{t}_{\mathrm{I}}$, must be of $25 \mathrm{~ms}$ maximum, that is, 40 steps to simulate a second, see [6].

In order to simulate at least $1 \mathrm{~s}$ of a future scenario, to predict the dynamic situation, position and active safety margin of the car, (under certain hypothesis of inputs to the model, driver actions on steering and brakes) then the model must be processed 40 times faster than the real time condition, and all this to get just one trajectory. If the goal is to evaluate 7 , for instance, (see Fig. 1) in order to choose the best one, then the computation demand in real time using numerical methods of integration is multiplied even more, Fig.2 shows this computation timing scheme.

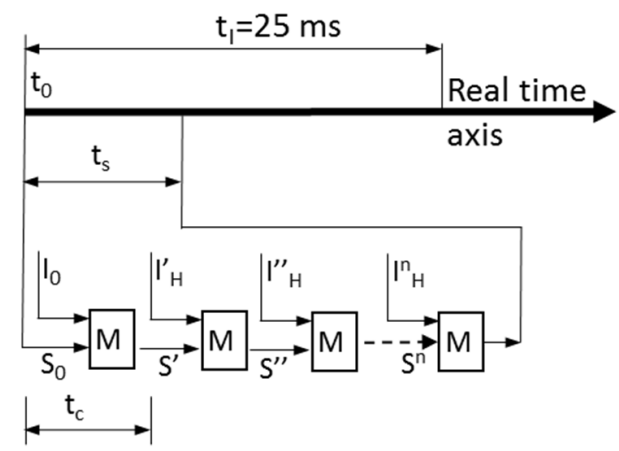

Fig. 2. Real time predictive simulation

In Fig. 2:

$\mathrm{t}_{\mathrm{c}}$ : It is the computing time required by the processing unit to simulate one computation step, solving the set of nonlinear differential equations of the vehicle dynamics plus a tyre model. $\mathrm{I}_{0}$ are the measured driver inputs to the model, braking and steering manoeuvers at the time $t_{0}$ when the predictive simulation starts. $\mathrm{S}_{0}$ is the set of states, initial conditions of speed (measured as well) in the $10 \mathrm{DOF}$ required to simulate. The successive states $S^{\prime}, S^{\prime \prime} \ldots S^{\text {n }}$ will not be measured anymore, but calculated as a result of the previous step, as it always happens in numeric simulation. To predict the position and dynamic situation of the car in the next one second, some hypothesis should be made about the possible driver actions during that second, they will be the hypothetic inputs I' ${ }_{\mathrm{H}}, \mathrm{I}{ }_{\mathrm{H}}$, $\ldots \mathrm{I}_{\mathrm{H}}^{\mathrm{n}}$. If the vehicle is "driver free" those inputs would be possible decisions considered by the computer to avoid the hazardous situation. At the end of those 40 steps, the computer has calculated (predicted) the position, dynamic situation and active safety margin of the car in the following second. To do this calculation, the computer has required $t_{\mathrm{s}}$ milliseconds of real time; being $t_{s}$ the simulation time. The condition for predictive simulation of just one trajectory $\left(\mathrm{t}_{\mathrm{s}}<\mathrm{t}_{\mathrm{I}}\right)$ is 40 times more demanding in terms of speed, than the real time condition $\left(\mathrm{t}_{\mathrm{c}}<\mathrm{t}_{\mathrm{I}}\right)$.

It is clear than the predictive simulation required for accident avoidance requires far more computation speed than the real time simulation.
Of course, the risk evaluation at the end of those 40 steps, in the following second should take into account the possible movements of the obstacles on the road, measured by advanced detection systems and some hypothesis of the maximum friction available in the tyre-road patch. A complex problem on the whole. Obviously, the requirement of computational power is tremendous.

Nevertheless, when observing the possible escape trajectories to avoid the collision without reaching situations of directional instability, leaving the road or wheel lock-up, it can be seen that the curves are smooth, and maybe they could be approximated with low degree polynomials avoiding those hundreds of thousands operations of the numerical methods.

The goal of this paper is to propose a methodology to obtain approximate solutions to the non-linear equations governing the behavior of a vehicle's dynamics in order to obtain a more efficient processing than the traditional numerical methods, appropriate for real time computation, that would facilitate the selection of evasive manoeuvers and avoid the collision.

Fig. 2 shows that if $t_{c}$ is reduced, the final simulation time $t_{s}$ will be reduced 40 times more.

In order to achieve this general goal, a significant reduction of the computation load, the approach of this paper is to forget the classic numerical integration methods and to approach the dynamic simulation in a different way.

To do this, different approximations and their application to vehicle decoupled models are analyzed to search for approximate polynomial analytical solutions to the dynamic equations.

The methodology in this paper is the following:

1.- As a general approach, symbolic computing is used instead of numeric.

2.- Search for pre-calculated solutions even though the systems are nonlinear, leaving for real time computing only a part of the computing load.

3.- Search for approximate solutions, accurate enough solutions instead of the exact ones.

This leads to series expansions of the differential equations (DE).

But to find them, the series expansions of functions (not DE) should be first analyzed, for two reasons:

- Because they are the base to the DE expansions.

- Because one of the conclusions of [6] was that more than a $50 \%$ of the computation time in the dynamic simulation of a car is due to the tyre model; for that reason this paper focus the series expansions of functions, on the algebraic equations depicting the tyre models. The most effective series expansions to approximate tyre models are rational functions, for that reason they must be included in the analysis, proposing a new tyre model much more efficient for real time applications, this is very important because it means a $50 \%$ of the computation time.

4.- Some examples of application are presented. 
TABLE I

METHODS AND TECHNIQUES

\begin{tabular}{|c|c|c|}
\hline \multicolumn{2}{|c|}{ TECHNIQUE-THEORY APPLIED } & COMMENTS \\
\hline \multicolumn{2}{|c|}{$\begin{array}{l}\text { Power series } \\
\text { Classic orthogonal functions } \\
\text { Families of orthogonal polynomials }\end{array}$} & $\begin{array}{l}\text { See bibliography. Not } \\
\text { detailed in this paper. }\end{array}$ \\
\hline \multicolumn{3}{|c|}{ II. APPROXIMATION OF FUNCTIONS } \\
\hline \multicolumn{2}{|c|}{ A.- Chebyshev Polynomials (ChP) } & Basis for the method \\
\hline \multicolumn{2}{|c|}{$\begin{array}{l}\text { B.- Approximation of functions through } \\
\text { Chebyshev Polynomials }\end{array}$} & $\begin{array}{l}\text { General method of } \\
\text { expansión of functions }\end{array}$ \\
\hline \multicolumn{2}{|c|}{$\begin{array}{l}\text { C.- Approximation using rational functions } \\
\text { Pade, Chebysheb-Pade, Remez. }\end{array}$} & $\begin{array}{l}\text { More accurate for tyre } \\
\text { models }\end{array}$ \\
\hline \multicolumn{2}{|c|}{$\begin{array}{l}\text { D.- Approximation using Jacobi } \\
\text { Polynomials }\end{array}$} & $\begin{array}{l}\text { More flexible. Used in } \\
\text { the new tyre model }\end{array}$ \\
\hline \multirow{2}{*}{\multicolumn{2}{|c|}{$\begin{array}{l}\text { E.- Examples of approximations of functions } \\
\text { applied to vehicle dynamics }\end{array}$}} & A new tyre model \\
\hline & & $\begin{array}{l}\text { Ackermann steering } \\
\text { mechanism }\end{array}$ \\
\hline \multicolumn{3}{|c|}{ III. APPROXIMATION OF DIFFERENTIAL EQUATIONS (DE) } \\
\hline \multicolumn{2}{|c|}{ A.- Expansion of a DE in power series } & Slow convergence \\
\hline \multicolumn{2}{|c|}{ B.- Expansion of a DE in Chebyshev series } & $\begin{array}{l}\text { Not used before in } \\
\text { automotive applications }\end{array}$ \\
\hline \multicolumn{2}{|c|}{ C.- Perturbation of differential systems } & $\begin{array}{l}\text { Fastest convergence } \\
\text { Polynomials of reduced } \\
\text { degree }\end{array}$ \\
\hline \multicolumn{2}{|c|}{ D.- Application example of perturbation } & Vertical dynamics \\
\hline $\begin{array}{l}\text { E.- Partially symbolic } \\
\text { solutions }\end{array}$ & \multicolumn{2}{|c|}{$\begin{array}{l}\text { Maximum flexibility. Allows to choose : } \\
\text { - Inputs to the model } \\
\text { - Model parameters } \\
\text { - Initial conditions } \\
\text { As the unknown variable allows obtaining } \\
\text { pre-calculated solutions }\end{array}$} \\
\hline \multirow{2}{*}{$\begin{array}{l}\text { Examples of partially } \\
\text { symbolic solutions }\end{array}$} & \multicolumn{2}{|c|}{ F.- Vertical model and 1 example } \\
\hline & \multicolumn{2}{|c|}{$\begin{array}{l}\text { G.-Longitudinal dynamics. Succesive } \\
\text { Approximations and } 4 \text { Case examples. }\end{array}$} \\
\hline
\end{tabular}

Table I shows a summary of the methods presented in this paper which have been applied to vehicle dynamics and a short comment about the reason or the main advantage of its use. The work flow of this paper follows the Table I.

More in detail, expansions in series based on the theory of approximation of functions, expansions in power series, and in Chebyshev and Jacobi series of orthogonal polynomials are analyzed, generally used in the theory of approximation; the convergence acceleration method of the power series, also called economization, is revised.

Then, the rational functions are introduced, providing a more accurate adjustment in multiple situations with a more efficient processing, for instance, in tyre models; Padé and ChebyshevPadé approximations, the Remez algorithm, and the Chebyshev and Jacobi expansions in series of Orthogonal Rational Functions (ORF's). This study includes the different quadrature algorithms that allow to obtain the series coefficients, for example, the Chebyshev-Gauss-Lobatto (CGL) quadrature, and its application to a suspension model is shown.
Subsequently, the approximation of differential equations is analyzed through the previous developments in series, and the theory of perturbation of dynamic systems allowing the reduction of the expansion polynomial degree is explained.

As general mathematical tool, the symbolic calculation program (MAPLE) is used, which becomes a true laboratory where results from the different techniques are compared with a systematic valuation of errors in the results achieved and of the speed in the series convergence. A test-error experimentation is carried out, testing the different techniques in every model, as the approximation is good depending on the shape of the curve to be approximated.

The previous approximation methods are applied to different models: tyre simulation, steering geometry, vertical and longitudinal dynamics, which serve to illustrate the use of the different described techniques while showing the huge reduction of computing time obtained when using approximate solutions.

Some application examples are presented.

The set of methods and techniques given here, never applied to vehicle dynamics before, may be used in more complex and integrated models as a follow-up of our research work.

\section{APPROXIMATION OF FUNCTIONS}

\section{A) Chebyshev Polynomials}

An introduction to power series, classic orthogonal function series and orthogonal polynomials can be found in [8], [9] and [10]. Now Chebyshev polynomials are briefly introduced as they are the base of all the approximation techniques and an essential element in the content of this paper.

Chebyshev polynomials [11], [12] of the first species are defined by:

$$
T_{n}(u)=\cos [n \cdot \arccos (u)]
$$

and fulfil the property of being orthogonal regarding the weight function $w(u)=\left(1-u^{2}\right)^{-1 / 2}$ in the interval $[-1,1]$.

The independent variable has to work, usually, in different orthogonality intervals $[a, b]$, for instance, in dynamic systems, the variable is time $t$ and will vary between the simulation's initial time and final time [tin, tfin]; then the following transformation will have to be performed:

$$
t=b \cdot u+c=\frac{t_{\text {fin }}-t_{\text {in }}}{2} \cdot u+\frac{t_{\text {fin }}+t_{\text {in }}}{2}
$$

The first Chebyshev polynomials are the following

$$
\begin{aligned}
& T_{0}(u)=1 ; T_{1}(u)=u ; T_{2}(u)=2 u^{2}-1 ; \\
& T_{3}(u)=4 u^{3}-3 u ; T_{4}(u)=8 u^{4}-8 u^{2}+1 \text { etc. }
\end{aligned}
$$

They can be computed and manipulated using MAPLE's Orthopoly library. In [11] formulae to obtain integrals, derivatives and products of Chebyshev polynomials can be found. 
B) Approximation of a Function through Chebyshev Polynomials

The continuous-infinite expansion of a function in Chebyshev series is as follows [11]:

$$
f(u)=\sum_{n=0}^{\infty} \alpha^{\prime}{ }_{n} T_{n}(u)
$$

where the single quote mark of the summation indicates the first term must be divided by two. The coefficients take the value:

$$
\alpha_{n}=\frac{2}{\pi} \int_{-1}^{1}\left(1-u^{2}\right)^{-1 / 2} f(u) T_{n}(u) d u
$$

When truncating the series at the $N$ degree, an approximation to the function is obtained, the more accurate the greater $N$ is. The truncation at a degree $\mathrm{N}-1$ is the best approximation to the $\mathrm{N}$ degree Chebyshev Polynomial. This is called economization and it will used at Table IV several times.

If $f(u)$ is a polynomial, then a closed form solution for the integral (5) can be found. This will allow to convert normal polynomials to Chebyshev polynomials. It is an exception, as usually for other non polynomial functions $f(u)$, quadrature formula must be used. In this cases, a Chebyshev discrete expansion can be used as seen in [13], for example applied to the approximation of the involute function in [14].

\section{C) Rational Functions}

Rational functions are essential when approximating functions with areas of sudden changes, for instance, those appearing in tyre models. In this case, the direct expansion in Chebyshev series does not converge properly and it is necessary the use of rational functions. That is the reason why they are included in this section, as they are an essential element in the proposed methodology. A rational function $r(x)$ with a $N$ degree looks this way

$$
r(x)=\frac{p(x)}{q(x)}=\frac{p_{0}+p_{1} x+\cdots+p_{n} x^{n}}{q_{0}+q_{1} x+\cdots+q_{m} x^{m}}
$$

Where $p(x)$ and $q(x)$ are the polynomials whose degrees add $N=n+m$. A rational approximation of degree $\mathrm{N}$ at the numerator and $\mathrm{M}$ in the denominator will be denoted as $[\mathrm{N}, \mathrm{M}]$

Padé rational approximations [9] allow the achievement of rational expressions with numerator and denominator developed in power series.

The Chebyshev-Padé developments allow to obtain more compact and exact rational expressions with Chebyshev polynomials both at the numerator and the denominator; they get good approximations but not those of a minimum maximum error (the so-called minimax). In order to find them, the Remez algorithm is used [15], [16] and [13], which stems from the Chebyshev-Padé approximation and tunes the result using numerical iterations converging in better approximation for both rational and polynomial functions. The justification and theoretical basic principles of these methods can be found in any book on the theory of approximation [17]. This Remez algorithm is implemented in MAPLE's Numapprox library with the minimax function.
In [18] and [19], the theory of orthogonal rational functions can be found, which allows the expansion of functions in series of rational functions. The authors of this article, have found a very efficient approximation of the magic formula tyre model expanding that formula in series of Jacobi polynomials of ORF's, (see II.E.1).

\section{D) Series of Jacobi Polynomials}

Within the families of classic orthogonal polynomials generated from the Sturm-Liouville differential equation, from which Chebyshev polynomials also derive, Jacobi polynomials $\operatorname{Pn}(x)$ are now considered, see [10]. They are orthogonal in the interval $[-1,1]$. The weight function in this type of polynomial is the following:

$$
\begin{aligned}
& w(x)=(1-x)^{\delta} \cdot(1+x)^{\gamma} \\
& \text { where } \delta \text { and } \gamma>-1
\end{aligned}
$$

The two parameters $\delta$ and $\gamma$ allow choosing the area of a best approximation at the orthogonality interval. This will allow us to improve the adjustment of the error at any area of the curve, for instance, to obtain a cero error at the origin of the interval or at the end.

Jacobi polynomials can also be computed and manipulated using the MAPLE Orthopoly library.

The expansion of a function in series of Jacobi polynomials uses the same expression (4), but using a Jacobi weight function this time. The integral must be programmed because a library for expansions of functions in Jacobi series is not available.

\section{E) Application examples \\ 1.- Example 1.- An efficient tyre model.}

Tyre behavior is a major factor of influence in active safety, see for example [20], [21] and [22]. Besides, according to [6], more than a $50 \%$ of the computing time in a vehicle dynamic simulation is due to tyre modelling. A lot of tyre models can be found in bibliography. One of the most accurate and widely used is the the well known model of Bakker, Nyborg and Pacejka, see [23], [24] and [25], is a semi-empiric tyre model based on the so called "magic" formula:

$$
Y=D \cdot \sin [C \cdot \arctan (B X-E \cdot[B X-\arctan (B X)])]
$$

The shape of the curve is controlled by the four parameters $B$, $C, D$ and $E$. The equation can calculate:

- Longitudinal force $F y$ and lateral force $F x$ as a function of longitudinal slip $K$ (in \%) and slip angle of the tyre $\alpha$, (in degrees) respectively.

- Self-aligning torque $M z$ also as a function of slip angle $\alpha$.

$B, C, D$ and $E$ constants describe: the inclination of the curve at the origin $(B C D)$, the peak value $(D)$, the curvature $(E)$ and the basic form $(C)$ for each case (lateral, braking or selfaligning torque). 
In [26], rational approximations and approximations in Jacobi series of ORF's to this magic formula have been presented. They are degree 3 polynomials and they simplify the model drastically with very low errors, $<1 \%$, allowing the calculation of both longitudinal and lateral forces in a computing time that divides by 20 those of the original formula (a 95\% computing time saving); the resulting final model is:

$$
f(x)=C_{0}+C_{1}\left(\frac{x}{x+b}\right)+C_{2}\left(\frac{x}{x+b}\right)^{2}+C_{3}\left(\frac{x}{x+b}\right)^{3}
$$

The optimal factor $b$ in each case varies with $F z$. As an example it is mentioned the original formulation in the computation of the longitudinal forces:

$$
F x=1122.7 \cdot \sin (1 \cdot 65 \cdot \arctan (0.0644 \cdot x+0.536 \cdot \arctan (0.138 x)))
$$

And the approximation obtained through expansion in ORFs Jacobi polynomial series:

$$
\begin{gathered}
\text { Fxap }=0.07759+(1238.28+(3235.35-3866.727 . v) \cdot v) \cdot v \quad ; \\
\text { being } v=x /(x+5.5)
\end{gathered}
$$

which is an outstanding effective processing polynomial with a maximum error $<1 \%$ regarding the original equation, as it can be seen in Fig. 3, where the curves practically overlap. Fig 4 , shows the error between both models. Obviously, the polynomial model has highly advantageous analytical properties: they can be evaluated, derivated and integrated very easily and the abscissa of maximum value can be computed with no difficulties.

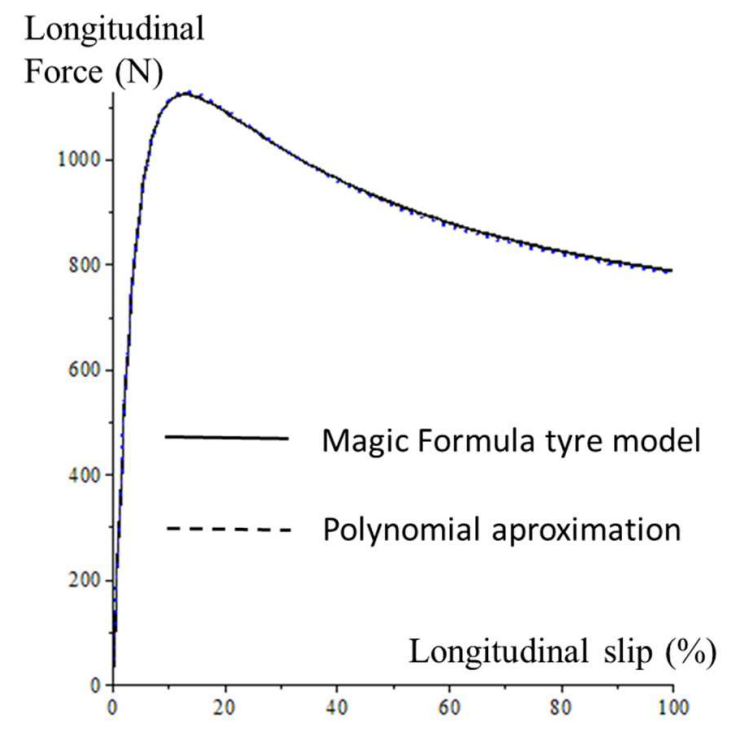

Fig. 3. Jacobi aproximation of the magic formula tyre model.

In the real time simulation, as explained in section I, the computing time can be reduced drastically using this polynomial model (95\% of saving) obtained by series expansion of the original Magic Formula, giving more free time for data acquisition, simulation, risk evaluation or decision making on real time.

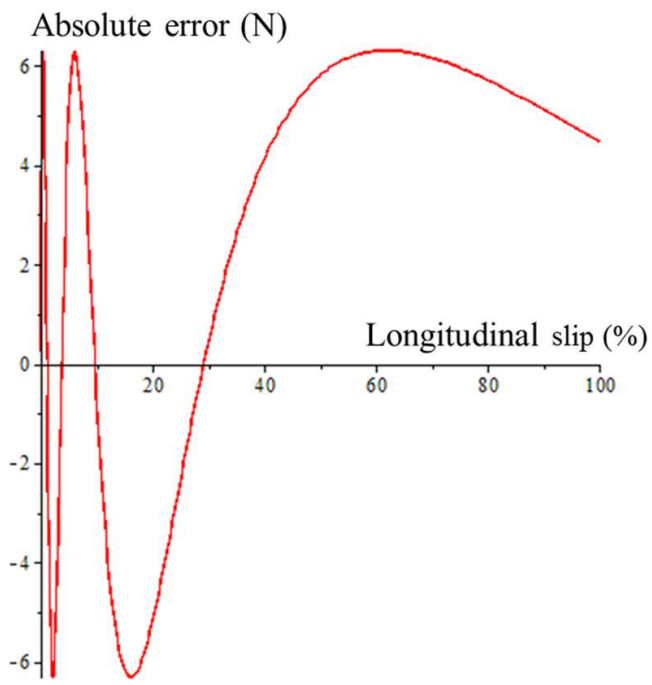

Fig.4. Absolute error in the aproximation of the magic formula tyre model $(\mathrm{N})$.

\section{2.- Example 2. Approximation of the Ackermann Geometric Condition of a Steering Mechanism}

The well known Ackermann condition relates the steering angle of the two interior and exterior wheels so that the perpendicular to the median plane of the wheel passes exactly through the same point $\mathrm{I}_{0}$, the instantaneous centre of rotation. The difference of the cotangents of both angles has to be the equal to the quotient between the track $v$, and the wheelbase of the vehicle $b$.

$$
\operatorname{cotg} \delta_{\text {ext }}-\operatorname{cotg} \delta_{\text {int }}=\frac{v}{b}=Z
$$

Given a steering angle value $\delta_{\text {int, }}$, the exterior angle is calculated with the expression:

$$
\delta_{\text {ext }}=\arctan \left(\frac{\tan \left(\delta_{\text {int }}\right)}{1+Z . \tan \left(\delta_{\text {int }}\right)}\right)
$$

Expanding the previous equation in Jacobi polynomials $[1,1]$ of rational functions, the following approximations can be obtained:

$$
\delta_{\text {ext }} \simeq \frac{\delta_{\text {int }}}{a_{1}+a_{2} \cdot \delta_{\text {int }}}
$$

As a numeric example, the data of a vehicle with a maximum turn of $40^{\circ}$ are shown, with the following values of wheelbase and track: $b=2700 \mathrm{~mm} ; v=1515 \mathrm{~mm} ; Z=0.561 ; a^{\prime} l=2.1547$; $a^{\prime} 2=2.21 ; d=-1 / 2 ; \gamma=-1$ and a relative maximum error $\varepsilon_{\text {rel }}$ max $=2.5 \%$ and an absolute maximum error $\varepsilon_{a b s \max }=15 \mathrm{E}-4$ $\mathrm{rad}=0.0859^{\circ}=0^{\circ} 5,15^{\prime}$.

Fig. 5 shows both curves, Ackerman's exact and approximate which almost overlap, altogether with the bisector corresponding to the parallel steering.

Evidently, as a' ${ }_{1}$ and a' ${ }_{2}$ are similar, it could be assumed that they are the same, generating the following ultra-compact expression:

$$
\delta_{\text {ext }} \simeq \frac{a_{1}^{\prime} \delta_{i n t}}{a_{\prime}^{\prime} 1+\delta_{\text {int }}}=\frac{\delta_{\text {int }}}{1+a_{3} \cdot \delta_{\text {int }}}
$$

$a^{\prime}{ }_{1}=a^{\prime}{ }_{2}=1.949 ; \quad a_{3}=1 / a^{\prime}{ }_{1}=0.513 ; \quad d=-1 / 2 ; \gamma=-1$; $\varepsilon_{\text {rel } \max }=0.85 \%$ and $\varepsilon_{a b s \max }=44 \mathrm{E}-4 \mathrm{rad}=0.252^{\circ}=0^{\circ} 15,12^{\prime}$ 


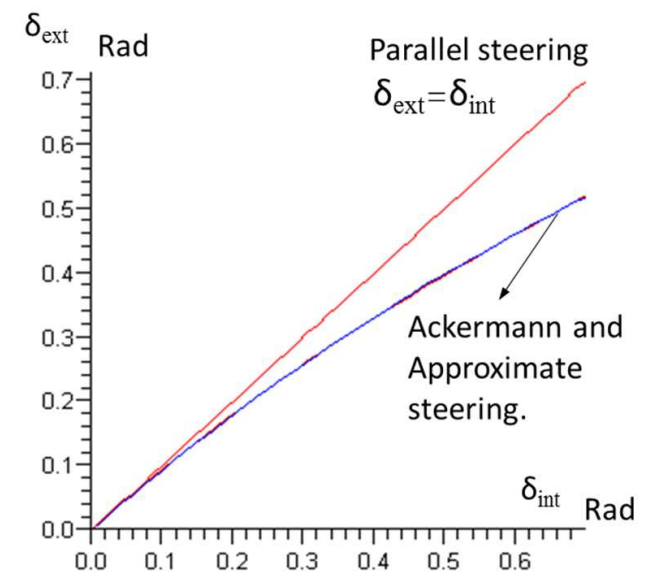

Fig. 5. Approximation of the steering angles.

These errors can be assumed or not depending on the application. As the quotient $Z=0.561$ of this vehicle is a very common value, it can be accepted that these approximations are valid for a wide set of cars.

Obviously, these approximated expressions have a much more efficient processing than the original formulation. The time loops tested show a processing 140 times faster than the initial Ackermann equation.

Chebyshev rational expansion in series $[1,1]$ generates lower absolute errors than Jacobi's; it is the particular case of $d=\gamma=-$ $1 / 2$; but it does not have a null error at the origin, which would imply that in the straight line the inner wheel has a zero angle, but the exterior one is turned permanently $12 \mathrm{E}-4 \mathrm{rad}=0^{\circ} 4,12^{\prime}$, which cannot be assumed conceptually.

$$
\delta_{\text {ext }} \simeq \frac{0.0010538+0.838219 . \delta_{\text {int }}}{0.866565+0.38226 . \delta_{\text {int }}}
$$

Chebyshev expansion in series [2,0] does not pass either through the origin and has an absolute error of $\varepsilon_{a b s \max }=24 E-4$ $\mathrm{rad}$, (the double of a rational one).

Expanding in Jacobi series [2,0] with a null error at the origin, the maximum error is $\varepsilon_{a b s \max }=32 E-4 \mathrm{rad}$, more than the double than Jacobi rational $[1,1]$ with the same number of parameters, 2.

The Taylor series expansion converges very slowly and, in its 2 degree expansion, presents an absolute error $\varepsilon_{\text {abs } \max }=950 \mathrm{E}-$ $4 \mathrm{rad}=5^{\circ} 26.4^{\prime}$ absolutely inadmissible, and in the 3 degree one an error $\varepsilon_{\text {abs max }}=127 \mathrm{E}-4 \mathrm{rad}=0^{\circ} 43.65^{\prime}$, which is still around 10 times greater than the Jacobi rational expansion of degree $[1,1]$.

Of course, the Chebyshev rational expansion [2,2] generates much lower errors: $\varepsilon_{\text {abs } \max }=17.5 \mathrm{E}-6 \mathrm{rad}=0^{\circ} 0$ ' 3.6", but with a bit more of computation.

Table 2 shows a summary of the previous approximations, indicating the error and the number of Floating Point Operations (FLOP). The ORF's [1,1] Jacobi expansion in series is the one generating the fastest convergence and the most compact expressions with the most reduced errors, the error passes through the origin.
TABLE II.

ApPROXIMATIONS OF THE ACKERMAN'S CONDITION

\begin{tabular}{|c|c|c|}
\hline Expansion & $\begin{array}{l}\text { Absolute error Rad x } \\
10 \mathrm{E}-4(\mathrm{grad})\end{array}$ & FLOP \\
\hline $\begin{array}{l}\text { Taylor } 2 \\
\qquad \delta_{\text {ext }} \approx \delta_{\text {int }}-K . \delta_{i n t}^{2}\end{array}$ & $950\left(5^{\circ} 26,4^{\prime}\right)$ & 3 \\
\hline $\begin{array}{l}\text { Taylor } 3 \\
\delta_{\text {ext }} \approx-K . \delta_{\text {int }}^{2}+K^{2} . \delta_{\text {int }}^{3}\end{array}$ & $127\left(0^{\circ} 43,65^{\prime}\right)$ & 8 \\
\hline $\begin{array}{l}\text { Chebyshev [2,0] } \\
\delta_{\text {ext }} \approx a_{3}+a_{4} \cdot \delta_{\text {int }}+a_{5} \cdot \delta_{\text {int }}^{2}\end{array}$ & $24\left(0^{\circ} 8,24^{\circ}\right)$ & 5 \\
\hline $\begin{array}{l}\text { Jacobi }[2,0] \\
\quad \delta_{\text {ext }} \approx a_{4} \cdot \delta_{\text {int }}+a_{5} \cdot \delta_{\text {int }}^{2}\end{array}$ & $32\left(0^{\circ} 11^{\prime}\right)$ & 4 \\
\hline $\begin{array}{l}\text { Chebyshev }[1,1] \\
\qquad \delta_{\text {ext }} \approx \frac{a_{3}+a_{4} \cdot \delta_{\text {int }}}{a_{1}+a_{2} \cdot \delta_{\text {int }}}\end{array}$ & $12\left(0^{\circ} 4,12^{‘}\right)$ & 5 \\
\hline $\begin{array}{l}\text { Jacobi }[1,1] \\
\qquad \delta_{\text {ext }} \approx \frac{\delta_{\text {int }}}{\delta^{\prime}}\end{array}$ & $15\left(0^{\circ} 5,15^{\prime}\right)$ & 3 \\
\hline $\begin{aligned} & a_{1}+a_{2} \cdot \delta_{i n t} \\
\delta_{\text {ext }} \simeq & \frac{\delta_{\text {int }}}{1+a_{3} \cdot \delta_{\text {int }}}\end{aligned}$ & $44\left(0^{\circ} 15,12^{\prime}\right)$ & 3 \\
\hline $\begin{array}{l}\text { Chebyshev [2,2] } \\
\delta_{\text {ext }} \approx \frac{a_{2}+a_{3} \cdot \delta_{i n t}+a_{4} \cdot \delta_{i n t}^{2}}{a_{5}+a_{6} \cdot \delta_{i n t}+a_{7} \cdot \delta_{i n t}^{2}}\end{array}$ & 0.17 (0 0'3.6”) & 11 \\
\hline
\end{tabular}

The use of those formulae can also save time during the car simulation, as explained in section I (the computing time is divided by 140). Starting from the steering wheel angle, we must obtain both angles in the inner and outer wheels related by the Ackermann condition to use them as inputs for simulation.

Some additional uses of those formulae can be done in other environments, for example to simplify the equations governing the Ackerman steering robots, for real time situations, see for example [27].

Once analysed the expansion of functions in Chebyshev series, the expansion of differential equations is introduced.

\section{APPROXIMATION OF DIFFERENTIAL EQUATIONS}

\section{A) Expansion of a Differential Equation in Power Series}

Although expansions in power series converge in a slower way than Chebyshev's or Fourier's, the authors of this article explored their application to active safety allowing to calculate the stopping distance and braking force required to stop the car in a given distance using very compact expressions and avoiding numerical simulations, see [28]. On the other hand, as the composing of differential equations in power series is the same as in Chebyshev series, it is shown as an example of the technique used. The power series method to resolve a differential equation consists of substituting the power series:

$$
y=\sum_{n=0}^{\infty} c_{n} x^{n}
$$

in the differential equation, previously derivated, to determine later the coefficient values $c_{0}, c_{1}, c_{2}, \ldots$, equating the expansion term by term so that the power series fulfils the differential equation. When truncating the series at a certain degree (for 
instance, 4) a certain error is generated. The expansion degree can be reduced by economization (see II.B).

Before substituting the power series (14) in a differential equation, derivatives $y^{\prime}, y^{\prime}$ "...can be calculated with a simple procedure consisting of writing the addition of the derivatives of the individual terms in the series for $y$.

\section{B) Expanding a Differential Equation (DE) in Chebyshev Series.}

The same methodology used in the development of power series can be applied now, but first it should be changed the domain from $t$ to $u$, which works in the interval $[-1,1]$, as seen in (2), and modify the DE accordingly. Expanding in terms of the speed, we get:

$$
x^{\prime}(u)=\sum_{i=0}^{N}{ }^{\prime} C_{i} \cdot T_{i}(u)
$$

expressions of $x "(u)$ and $x(u)$, can be obtained integrating and deriving the expression of $x^{\prime}(u)$.

Now the differential equation can be composed, expanding each Chebyshev polynomial and collecting it in powers of $u$ according to the identity principle. In order to fulfil the equality all the coefficients of the expansion in $u^{i}$ must be identically null, which generates a system of equations whose solution calculates the coefficients of the three expansions $x^{\prime \prime}(u), x^{\prime}(u)$ and $x(u)$. It can be improved a lot the accuracy of those solutions, for the same degree of polynomial, using the so called perturbation methods, as follows:

\section{C) Perturbation of differential systems.}

This theory was initially developed by Lanczos, see [29] and [11] for applications. This theory states that if the finite expansion of an equation in Chebyshev series approximately fulfils the integrated equation of an ODE, it is possible to find a small perturbation term of that equation, so that the expansion of $y(u)$ exactly fulfils the perturbed dynamic equation. The number of perturbation terms depends on the number of non fulfiled equations resulting from the truncation of the series at $\mathrm{N}$ degree when equalling term by term the resulting Chebyshev series coefficients of the complete assembly of the dynamic equations. Using perturbation terms, the degree of the expansion, or the error for the same degree can be strongly reduced (see Fig. 7-8).

\section{D) Application examples}

\section{1.- Example 3.- Expanding the Vertical 1 DOF Model in} Chebyshev Series.

The 10 DOF model mentioned in section I includes 4 coupled vertical suspension submodels; for that reason, the decoupled 1 DOF model is expanded in Chebyshev series.

In the simplest case of the 1 DOF vertical model, (see Fig.6), when expanding in Chebyshev series the differential equation, the set of perturbed equations is the following:

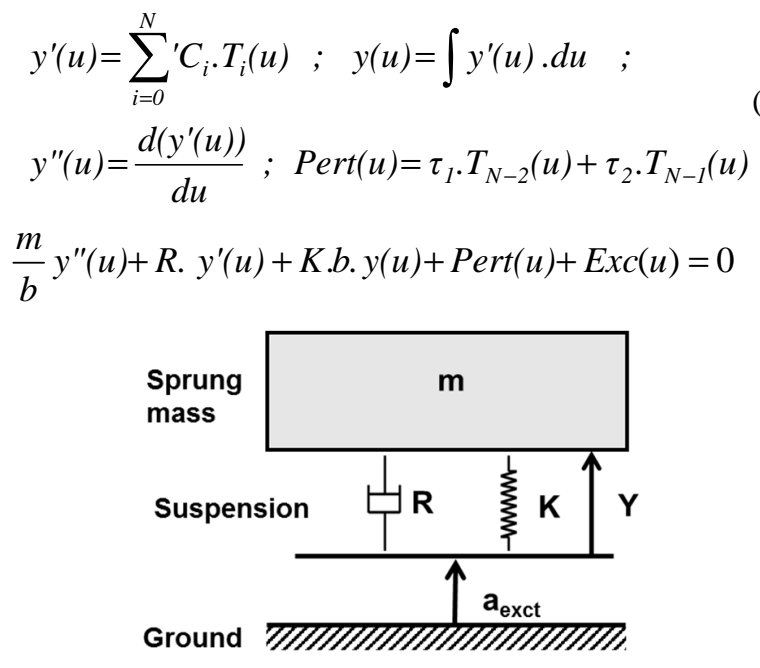

Fig. 6. 1 DOF suspension model.

For example, assuming $K=12.000 \mathrm{~N} / \mathrm{m}, R=800 \mathrm{Ns} / \mathrm{m}$ and $m=300 \mathrm{~kg}$, with the initial conditions, $y_{0}=0.1, v_{0}=0.1, t_{\text {fin }}=1.5$ $s$ and the following excitation $E x c=2 . h . t^{3} / a^{3}+3 . h \cdot t^{2} / a^{2}$, with $a=3$ and $h=2000$, with an expansion of degree 11 , figures 7 and 8 show the difference between applying or not the perturbation terms:

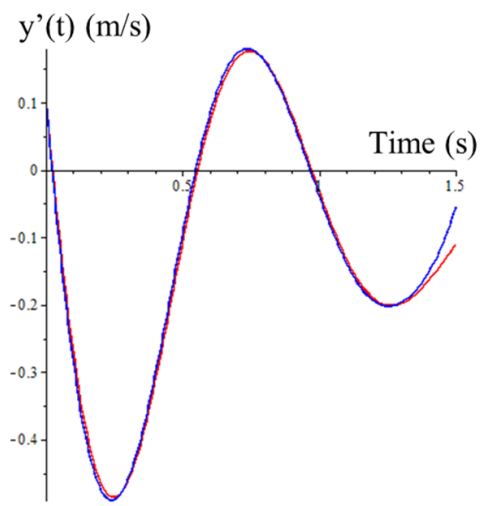

Fig. 7. Vertical speed $y^{\prime}(t)$ in the vertical 1 DOF model without perturbation terms.

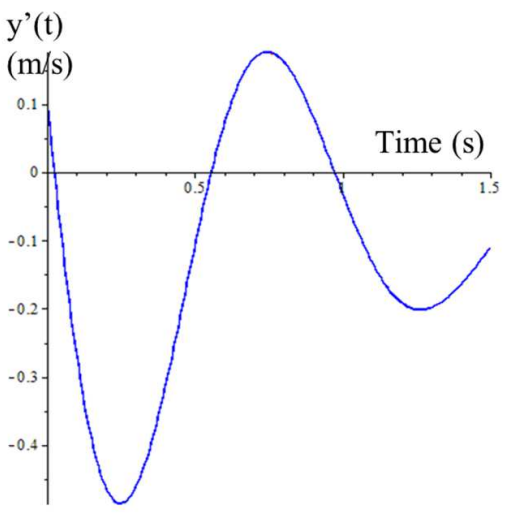

Fig. 8. Vertical speed $y^{\prime}(t)$ in the vertical 1 DOF model with perturbation terms.

The solution of this vertical model, for example with an expansion of degree 5 in terms of $u$ is: 
$v(u) \approx \frac{C_{0}}{2}+C_{1} \cdot T_{1}(u)+C_{2} \cdot T_{2}(u)+C_{3} \cdot T_{3}(u)+$

$C_{4} \cdot T_{4}(u)+C_{5} \cdot T_{5}(u)$

The maximum errors in this case are $0.05 \mathrm{~m} / \mathrm{s}$ without perturbation terms and $0.00002 \mathrm{~m} / \mathrm{s}$ with perturbation.

Converting the expansion (17) in a normal polynomial substituting the Chebyshev Polynomials $T_{i}(u)$ for its values, see (3), a polynomial solution of the vertical speed is obtained. Some interesting applications can be found if the values of some parameters are not assigned, let's see it.

\section{E) Partially symbolic solutions}

If some of the values of the different model parameters, initial conditions or the excitation are not assigned, mixed or partially symbolic solutions can be obtained. The numerical part of the solution is pre-calculated off-line and the symbolic part is calculated on real time, in function of the inputs to the model, measured on real time, let's see.

\section{F) Solution of the vertical model with initial conditions $\left(y_{0}, v_{0}\right)$, excitation and parameters $(m, K$ or $R)$ not defined}

If the vertical force of excitation is a cubic spline:

$E x c=C_{f 0}+C_{f 1 .} . t+C_{f 2} . t^{2}+C_{f 3} . t^{3}$, being known $K, m$ and $R$, the coefficients of the Chebyshev series representing the system response have the following form:

$$
\begin{aligned}
& C_{i}=P_{v 0 i} \cdot v_{0}+P_{y 0 i} \cdot y_{0}+P_{E X C i 0} \cdot C_{f 0}+ \\
& +P_{E X C i 1} \cdot C_{f 1}+P_{E X C i 2} \cdot C_{f 2}+P_{E X C i 3} \cdot C_{f 3}
\end{aligned}
$$

Where $P_{v o i}, P_{y o i}, P_{E X C i j}$ are numerical coefficients.

In [30] it is fully explained the development in Chebyshev series the suspension model of $2 \mathrm{DOF}$ of a quarter of a vehicle, obtaining very compact polynomial solutions regarding the initial conditions and excitation, (being known all the parameters of the model). The expression of the general term is presented and it is also shown the enormous improvement in the computational efficiency obtained by means of this method, with low frequency vertical excitations.

But this paper goes a step beyond, leaving undefined the model parameters too. This can be useful, particularly with the variation of the vehicle mass, which depends on the load of the vehicle and it is not a fixed parameter. It should be estimated when the trip begins.

If, in addition to the excitation Exc and initial conditions $\left(y_{0}, v_{0}\right)$, neither one of the three parameters (only one) is defined $K, m$ or $R$, then $P_{v o i}, P_{y o i}, P_{E X C i j}$ in (22) are not constant anymore, but they are rational functions in the undefined parameter ( $m, K$ or $R$ ). The maximum degree of those rational functions is $[N, N]$, depending on the order of the coefficient and on which parameter is undefined. $N$ is the degree of the expansion.

Those rational functions can still be approximated to simple polynomials of degree 2 or 3 at most, in function of the undefined parameter using the Minimax-Remez algorithm,

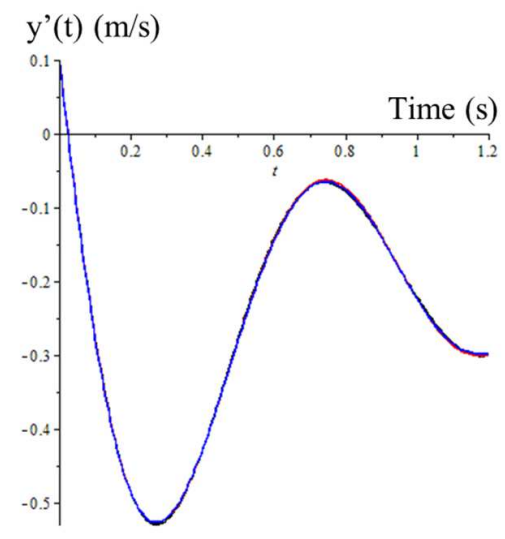

Fig. 9. Vertical speed $y^{\prime}(t)$ in the vertical 1 DOF model with $\mathrm{m}$ approximated from $[6,6]$ to $[2,0]$.

with a very low error $(<1 \%)$. If the undefined parameter is the mass $m$ for example, maximum and minimum values for $m$ have to be defined, that is $250 \mathrm{~kg}$ and $400 \mathrm{~kg}$ and shift the Chebyshev polynomials to the orthogonality interval [-1..1] as explained in equation (2).

This method, inserts the theory of approximation into the series resolution of the differential equation.

1.- Example 4.- Obtaining a pre-calculated response of a vertical 1 DOF model in function of initial conditions, excitation and mass.

Model of $1 \mathrm{DOF}$, forced vibration. Mass $m$, initial conditions $y_{0}, v_{0}$ and the excitation Exc, are now undefined:

In this case, the initial conditions $y_{0}, v_{0}$ can be measured in real time using sensors of displacement in the suspension, also the road profile (and its associated vertical excitation) can be a function of the car situation (obtained with a GPS for example) or it can also be measured using laser sensors. Finally, the mass depends on the load and it can be estimated when the trip begins, using the same displacement sensors. The vertical response of the model is a pre-calculated polynomial function of those variables, measured in real time $y_{0}, v_{0}, m$, and $C_{f i}$ and the final response can be calculated very fast by means of equations (17) and (18).

$E x c=C_{f 0}+C_{f 1} \cdot t+C_{f 2} \cdot t^{2}+C_{f 3} \cdot t^{3} ;$ being $C_{f 0}=0 ; C_{f 1}=0$;

$C_{f 2}=3 . h / a^{2} ; C_{f 3}=-2 . h / a^{3} ; a=1.5 ; h=3000$.

Expanding until $t_{\text {fin }}=1.2 \mathrm{~s}$ with a degree of the expansion $N=6$, with $R=800$ and $K=12000$.

The coefficients $P_{v o i}(m), P_{y o i}(m)$, and $P_{E X C i j}(m)$ can be approximated using a Minimax-Remez [2,0] assuming $250 \mathrm{~kg} \leq m \leq 400 \mathrm{~kg}$.

Fig. 9 shows the results in speed, for $m=300, v_{0}=0.1 \mathrm{~m}^{-1} \mathrm{~s}^{-1}$ and $y_{0}=0.1 \mathrm{~m}$. The numerical calculation and the approximate one almost overlap.

The number of FLOP is 96, it can be considered that the numerical integration of the DE $m \cdot a(t)+R \cdot v(t)+K \cdot y(t)+E x c=0$ using the most simple (and fast) integration method (Euler), with integration step $=0.025 \mathrm{~s}$ (40 steps per second) and $t_{\text {fin }}=1.2 \mathrm{~s}$, requires at least $9 \times 48=432$ FLOP. Using the 4-step 
Runge-Kutta method, then number of FLOPS is approximately 4 times bigger (1728 FLOP).

Even though the preparation of the approximation presented here is obviously more complex than the numerical integration, the real time execution is much faster. Being as the mass $m$ of a car changes only once every trip, then $P_{v o i}(m), P_{y o i}(m)$, and $P_{\text {EXCij }}(m)$ are constant and the number of FLOP is reduced to 24 instead of 96.

Different areas of application could be explored as well:

- Diagnostics and parameter estimation; for example leaving undefined the damping coefficient $\mathrm{R}$, it can be easily obtained the expected response in function of $R$ and compare it with the measured responses for given values of mass and excitation, using Kalman filters and estimating in this way the state of the damper.

- Control of semi-active suspension systems. The response of the suspension for different values of $\mathrm{R}$, can be calculated extremely fast using (17) and (18), adapting it to every excitation frequency and avoiding numerical simulation.

\section{G) Symbolic Solution of the longitudinal model}

Now the longitudinal dynamics model of a vehicle is analysed, performing an approximation of the whole model. The nonlinear equation describing the longitudinal dynamics of a car is the following:

$$
\begin{aligned}
& m \cdot\left(\frac{d^{2} x}{d t^{2}}\right)+K_{a} \cdot\left(\frac{d x}{d t}\right)^{2}+K_{1} x+K_{0}+K_{r}+E x c=0 ; \\
& E x c=C_{f 3} t^{3}+C_{f 2} t^{2}+C_{f 1} t+C_{f 0}
\end{aligned}
$$

Table III shows the notation:

TABLE III

TERMS OF THE LONGITUDINAL DYNAMIC EQUATION.

\begin{tabular}{|l|l|}
\hline$m$ & $\begin{array}{l}\text { Vehicle mass equivalent, } \\
\text { including the rotating masses } \\
\text { effect }\end{array}$ \\
\hline$K_{a}=\frac{1}{2} \cdot C_{x} \cdot A_{f} \cdot \rho$ & $\begin{array}{l}\text { Longitudinal aerodynamic } \\
\text { resistance coefficient }\end{array}$ \\
\hline$C_{x}$ & $\begin{array}{l}\text { Frontal area of the vehicle } \\
A_{f}\end{array}$ \\
\hline$\rho$ & $\begin{array}{l}\text { Air density } \\
\text { Rolling resistance force }\end{array}$ \\
\hline$f_{r}=m \cdot g \cdot f_{r}$ & $\begin{array}{l}\text { Rolling resistance coefficient } \\
f_{r}\end{array}$ \\
\hline$P \cdot \sin (\alpha) \approx P \cdot \tan (\alpha)$ & $\begin{array}{l}\text { Gravitational force, assuming a } \\
\text { road with parabolic profile }\end{array}$ \\
\hline$P \frac{d y}{d x}=K_{1} \cdot x+K_{0}$ & $\begin{array}{l}\text { Braking force, as a } \\
\text { cubic spline }\end{array}$ \\
\hline$E x c=C_{f 3} \cdot t^{3}+C_{f 2} \cdot t^{2}+C_{f 1} \cdot t+C_{f 0}$
\end{tabular}

In order to find the approximate solution of the equation (19), the most efficient approach is the use of symbolic successive approximations, with only one iteration. It produces low errors $(<1 \%)$ for short intervals of time ( 2 or $3 \mathrm{~s}$ ), with a number of operations quite reduced.

The first approximation to the speed is the following:

$$
\begin{aligned}
& x_{1}(t)=x_{0}+\int v_{0} d t \\
& v_{1}(t)=v_{0}-\frac{1}{m} \int\left(K_{a} \cdot v_{0}^{2}+K_{1} \cdot x_{1}(t)+K_{0}+K_{r}+E x c\right) \cdot d t
\end{aligned}
$$

Being $x_{0}$ the initial condition of longitudinal displacement and $v_{0}$ the initial longitudinal speed.

The second approximation to the speed can be obtained from the previous:

$$
\begin{aligned}
& x_{2}(t)=x_{0}+\int v_{1}(t) d t \\
& v_{2}(t)=v_{0}-\frac{1}{m} \int\left(K_{a} \cdot v_{1}(t)^{2}+K_{1} \cdot x_{2}(t)+K_{0}+K_{r}+E x c\right) \cdot d t
\end{aligned}
$$

Taking $N=3$ initially. As in (21), the speed is squared, an approximation of degree 6 is obtained; it can be reduced to degree 3 truncating the Chebyshev series, obtaining a 100\% symbolic solution.

In the final step (calculation of $\mathrm{v}_{2}(\mathrm{u})$ ), the polynomial is raised to degree 4 due to the integration.

To approximate the series, the independent variable must work in the domain of $\mathrm{u}[-1 . .1]$. In the transformation $t=b . u+c$, (see (2)), it appears the Jacobian term $b$, because $d t=b . d u$. For that reason, the equations (20) and (21) have to be modified as follows:

$$
\begin{aligned}
& x_{1}(u)=x_{10 u}+b \int v_{0} d u=b \cdot v_{0} \cdot u+x_{0}+b \cdot v_{0}=v_{0} \cdot u+v_{0} \\
& v_{1}(u)=v_{10 u}-\frac{b}{m} \int\left(K_{a} \cdot v_{0}^{2}+K_{1} \cdot x_{1}(u)+K_{0}+K_{r}+\operatorname{Exc}(u) \cdot d u\right. \\
& x_{2}(u)=\left(x_{20 u}+b \cdot \int v_{1}(u) d u\right) ; \\
& v_{2}(u)=v_{20 u}-\frac{b}{m} \int\left(K_{a} \cdot v_{1}(u)^{2}+b \cdot K_{1} \cdot x_{2}(u)+K_{0}+K_{r}+\operatorname{Exc}(u)\right) \cdot d u
\end{aligned}
$$

As the values of the initial conditions are known in $t=0, u=-1$, it happens that $x_{10 u} \neq x_{20 u} ; v_{10 u} \neq v_{20 u}$. Considering a simulation between $t_{i n}=0$ and $t_{\text {fin }}=2$, then $b=c=1$. We will continue with those values to avoid drag them. An additional assumption is $x_{0}=0$. Table IV shows the "cascade" code.

The term "cascade" means that every term in Table IV is computed from the previous, following equations (22) and (23) in a descending work flow:

$$
\begin{aligned}
& x_{1}(u)=f\left(u, v_{0}\right) ; \\
& v_{1}(u)=f\left(u, x_{1}(u), v_{10 u}, K^{*}, \operatorname{Exc}(u)\right) ; \\
& x_{2}(u)=f\left(u, v_{1}(u), x_{20 u}\right) ; \\
& v_{2}(u)=f\left(u, x_{2}(u), v_{20 u}, v_{1}(u), K^{*}, \operatorname{Exc}(u)\right)
\end{aligned}
$$

Being $K^{*}$ the parameters of the model $\left(m, K_{a}, K_{l}, K_{0}, K_{r}\right)$. The calculation requires 186 FLOP.

The numerical integration of this nonlinear differential equation, using the Euler method requires 18 FLOP per integration step, about 1440 FLOP for $t_{f i n}=2 s$ with 40 steps every second. This method divides the computing time by 7.7 , saving the $87 \%$ of the calculation.

If $N=4$, then FLOP=221, obtaining an even better accuracy. An important advantage of this method is that the set of calculations shown at Table IV is fully symbolic, so the terms that will not change can be pre-calculated and the rest can be 
measured with sensors (braking actions, initial speed, slope etc.).

TABLE IV

APPROXIMATE CALCULATION OF V(t) USING SYMBOLIC SUCCESSIVE APPROXIMATIONS.

\begin{tabular}{|c|c|c|c|}
\hline \multicolumn{3}{|l|}{ Calculation } & FLOP \\
\hline$b, c$ & \multicolumn{2}{|l|}{$\begin{array}{l}b=\left(t_{\text {fin }}-t_{\text {in }}\right) / 2 ; c=\left(t_{\text {fin }}+t_{\text {in }}\right) / 2 ; \\
t_{\text {in }}=0 ; t_{\text {fin }}=2: b=c=1 ; N=3 ; x_{0}=0\end{array}$} & \\
\hline Auxiliary terms & \multicolumn{2}{|l|}{$\begin{array}{l}v_{02}:=v_{0}^{2} ; C_{f}=C_{f 0}+C_{f 1}+C_{f 2}+C_{f 3} ; \\
R_{0}=C_{f}+K_{0}+K_{l} v_{0}+K_{r o d}+K_{a} \cdot v_{02} ; r=1 / m\end{array}$} & 11 \\
\hline$x_{10 u} \quad$ in $(22)$ & \multicolumn{2}{|l|}{$x_{10}(u)=b \cdot v_{0}+x_{0}=v_{0}$} & \\
\hline$x_{l}(u)$ in $(22)$ & \multicolumn{2}{|l|}{$x_{l}(u)=v_{0} \cdot u+v_{0}$} & \\
\hline$v_{10 u} \quad$ in $(22)$ & \multicolumn{2}{|l|}{$\begin{aligned} v_{10 u}=v_{0} & -r .\left[2 \cdot\left(K_{a} \cdot v_{02}+C_{f 0}+K_{0}+K_{r o d}\right)\right. \\
& \left.+K_{1} \cdot v_{0}+C_{f 1}\right] / 2\end{aligned}$} & 11 \\
\hline$v_{l}(u)$ in $(22)$ & \multicolumn{2}{|l|}{$\begin{array}{l}v_{14 L}=-r \cdot C_{f 3} / 4 \\
v_{13 L}=-r \cdot\left(C_{f 2} / 3+C_{f 3}\right) \\
v_{12 L}=-(r / 2) \cdot\left(K_{1} \cdot v_{0}+C_{f 1}+2 \cdot C_{f 2}+3 \cdot C_{f 3}\right) \\
v_{11 L}=-r \cdot R_{0} \\
v_{10 L}=-r \cdot\left(C_{f 2} / 3+C_{f 3} / 4\right)+v_{10 u}\end{array}$} & 25 \\
\hline $\begin{array}{l}\text { Economization } \\
\text { of } \mathrm{v}_{1}(u) \text { from } 4 \\
\text { to } 3\end{array}$ & \multicolumn{2}{|l|}{$\begin{array}{l}v_{13}=v_{13 L} ; v_{12}=v_{12 L+} v_{14 L} \\
v_{11}=v_{11 L} ; v_{10}=v_{10 L}-v_{14 L} / 8 \\
v_{1}(u)=v_{10+} v_{11} \cdot u_{+} v_{12} \cdot u^{2}+v_{13}{ }^{3}\end{array}$} & 3 \\
\hline$x_{20 u} \quad$ in (23) & \multicolumn{2}{|l|}{$\begin{aligned} x_{20 u}= & r \cdot\left[\left(20 \cdot K_{1}+60 \cdot m\right) \cdot v_{0}+20 C_{f 1}+\right. \\
& \left.+27 \cdot C_{f 3}+25 \cdot C_{f 2}-30 \cdot R_{0}\right] / 60\end{aligned}$} & 14 \\
\hline \multicolumn{4}{|c|}{ Detail of next calculations ommited for clarity } \\
\hline \multicolumn{2}{|c|}{$x_{2}(u) \quad$ in $(23)$} & - & 4 \\
\hline \multicolumn{2}{|c|}{ Economization of $x_{2}(u)$ from 5 to 3} & - & 6 \\
\hline \multicolumn{2}{|c|}{$v_{l}(u)^{2}$} & - & 20 \\
\hline \multicolumn{2}{|c|}{ Economization of $v_{l}(u)^{2}$ from 6 to 3} & - & 11 \\
\hline \multicolumn{2}{|c|}{$v_{20 u} \quad$ in $(23)$} & - & 21 \\
\hline \multicolumn{2}{|l|}{$v_{2}(u)$ in $(23)$} & - & 34 \\
\hline \multicolumn{2}{|c|}{ Going back to the $t$ domain $\left[0 . . t_{\text {fin }}\right] ; t_{\text {fin }}=2$} & - & 26 \\
\hline \multicolumn{3}{|l|}{ TOTAL } & 186 \\
\hline
\end{tabular}

Thus, the solution indicated in Table IV can be drastically simplified if the goal is to represent the solution in function of one or two of its parameters for a more detailed analysis or fast computing.

1.-Example 5. Calculation of the speed and position of a car in the following seconds in function of a constant braking and initial speed.

Considering just a constant braking $E x c=C_{f 0}$ from an initial speed $\mathrm{v}_{0}$, the resulting speed $v(t)$ calculated in Table IV is now reduced to the code (24):

$v_{t 4}=n_{i j} \cdot v_{0}^{3}+n_{i j} \cdot v_{0}^{2}+\left(n_{i j} \cdot C_{f 0}+n_{i j}\right) \cdot v_{0}$

$v_{t 3}=n_{i j} \cdot v_{0}^{4}+\left(n_{i j} \cdot C_{f 0}+n_{i j}\right) \cdot v_{0}^{2}+n_{i j} \cdot\left(C_{f 0}+n_{i j}\right) \cdot\left(C_{f 0}+n_{i j}\right)$

$v_{t 2}=n_{i j} \cdot v_{0}^{3}+n_{i j} \cdot v_{0}^{2}+\left(n_{i j}+n_{i j} \cdot C_{f 0}\right) \cdot v_{0}$

$v_{t l}=n_{i j} \cdot v_{0}^{2}+n_{i j} \cdot C_{f 0}+n_{i j} ; \quad v_{t 0}=v_{0}$

$v(t)=v_{t 0}+v_{t 1} \cdot t+v_{t 2} \cdot t^{2}+v_{t 3} \cdot t^{3}+v_{t 4} \cdot t^{4}$

Where $n_{i j}$ are numerical coefficients (all different and precalculated). This calculation requires only 42 FLOP.

It is supossed that all the other parameters are fixed (constant slope, constant mass, aerodynamic drag etc.).

4- Example 6. Calculation of the speed and position of a car in the next 2 seconds in function of the initial speed given a fixed braking action.
As a particular case, the coefficients of the speed in function of $v_{0}$, for a given fixed braking action, take the very simple following form:

$$
v_{t i}=n_{i 0}+n_{i 1} \cdot v_{0}+n_{i 2} \cdot v_{0}^{2}+n_{i 3} \cdot v_{0}^{3}+n_{i 4} \cdot v_{0}^{4}
$$

2.- Example 7. Calculation of the speed and position of a car in the next 2 seconds in function of the braking action for a fixed initial speed.

$v(t)$ can be expressed in function of $C_{f 0}$, obtaining coefficients which are polynomials of degree 2. $v_{t i}=c_{i 0}+c_{i 1} . C_{f 0}+c_{i 2} . C_{f 0}{ }^{2}$.

3.- Example 8. Calculation of the minimum braking force required to stop a car running at $20 \mathrm{~m} / \mathrm{s}$ in 3 seconds on a descending slope of $6 \%$ under uncertain friction conditions.

In this example, the car $n^{\circ} 2$ is detected approaching our car $\left(\mathrm{N}^{\circ}\right.$ 1). In 3 seconds both trajectories could cut.

It could lead to a risky situation or not, depending on the next trajectory and speed evolution of the car $\mathrm{N}^{\circ} 2$. The electronic driver of our car $\mathrm{N}^{\circ} 1$ must be prepared for both scenarios, a possible crash or not. It must know if a braking maneuver could stop the car in 3 seconds but the friction coefficient between the road and tyres cannot be estimated in a very accurate way (as usual). It is not raining, the friction coefficient $\mu$ in a normal road could take values between 0.6 and 0.9 , which, for a normal passenger car, usually leads to maximum allowed decelerations between $0.6 \mathrm{~g}$ and $0.9 \mathrm{~g}$ and maximum braking forces (in the best case) between 0.6.M.g and 0.9.M.g (between $10594 \mathrm{~N}$ and $15892 \mathrm{~N}$ in our car).

Data:

Mass, $\mathrm{m}=1800 \mathrm{Kg}$. Measured when the trip begins using the suspension displacement sensors.

Aerodynamic drag $K_{\mathrm{a}}=\mathrm{C}_{\mathrm{x}} \cdot \mathrm{A}_{\mathrm{f}} \cdot \mathrm{\rho} / 2=0.5$ obtained from constant data of the vehicle (It could be tuned if a window is open).

Rolling resistance force $\mathrm{K}_{\mathrm{r}}=170 \mathrm{~N}$ (assumed constant).

Slope $=-6 \%$ obtained by means of the GPS which locates the exact situation of the car (assuming that the vertical profile of the road is also in the data base). It can be fusioned with body level sensors and suspension displacement sensors.

The equations (24) become:

$$
\begin{aligned}
v_{t 4}= & 9.91 E-11 \cdot v_{0}^{3}-1.83 E-9 \cdot v_{0}^{2}+\left(1.98 E-10 \cdot C_{f 0}+1.34 E-6\right) \cdot v_{0} \\
v_{t 3}= & -7.1 E-12 \cdot v_{0}^{4}+\left(-2.85 E-11 \cdot C_{f 0}-7.46 E-7\right) \cdot v_{0}^{2}- \\
& 2.8 E-11 \cdot\left(C_{f 0}+17885\right) \cdot\left(C_{f 0}+1229.5\right) \\
v_{t 2}= & 7.7 E-8 \cdot v_{0}^{3}-1.85 E-9 \cdot v_{0}^{2}+\left(0.0027+1.54 E-7 . C_{f 0}\right) \cdot v_{0} \\
v_{t l}= & -0.00027 \cdot v_{0}^{2}-0.00055 \cdot C_{f 0}-0.68 ; \quad v_{t 0}=v_{0} \\
v(t)= & v_{t 0}+v_{t t} \cdot t+v_{t 2} \cdot t^{2}+v_{t 3} \cdot t^{3}+v_{t 4} \cdot t^{4}
\end{aligned}
$$

These (26) are pre-calculated from the mass estimation when the trip begins.

Running on real time, our electronic driver knows the current speed of the car $\mathrm{v}_{0}=20 \mathrm{~m} / \mathrm{s}$, for this speed, the equations (26) become:

$$
\begin{aligned}
& v_{t 4}=3.96 E-9 . C_{f 0}+0.000027 \\
& v_{t 3}=-2.85 E-11 . C_{f 0}{ }^{2}-5.57 E-7 . C_{f 0}-0.00092 \\
& v_{t 2}=3.08 E-6 . C_{f 0}+0.0558 ; \\
& v_{t 1}=-0.00055 . C f 0-0.794 ; \quad v_{t 0}=v_{0} \\
& v(t)=v_{t 0}+v_{t 1} . t+v_{t 2} . t^{2}+v_{t 3} . t^{3}+v_{t 4} \cdot t^{4}
\end{aligned}
$$


22 FLOP have been used to obtain the new equations (27).

In $t=3, v$ becomes just a function of the constant braking force $C_{f 0}$ :

$$
v(C f O)=18.09-0.0016 . C f O-7.71 E-10 . C f O^{2}
$$

20 additional FLOP have been used to obtain (34) whose root is $\mathrm{C}_{\mathrm{f} 0}=10888.5 \mathrm{~N}$ which requires 6 additional FLOP plus a square root.

The result in this case indicates that the force required to stop our car in 3 seconds $(10888.5 \mathrm{~N})$ is very close to the lowest requirement of friction in the tyre-road contact patch (10594 $\mathrm{N}$ ) or in other words, the braking required deceleration would be $0.616 \mathrm{~g}=6.05 \mathrm{~m} . \mathrm{s}^{-2}$, in the upper limit of moderate braking manoeuvers. During normal driving conditions in an open road, decelerations between 0 and $3 \mathrm{~m} . \mathrm{s}^{-2}$ are normal, between 3 and $6 \mathrm{~m} . \mathrm{s}^{-2}$ only appear in risk or unexpected situations and beyond $6 \mathrm{~m} . \mathrm{s}^{-2}$ means that a high risk or accident situation is happening (or a hard sport driving). A car with ABS can easily reach such deceleration of $6 \mathrm{~m} \cdot \mathrm{s}^{-2}$ on dry asphalt.

58 FLOP plus a square root have been used in total on the real time calculation, that means few microseconds in an advanced on board microcomputer with floating point operations capacity.

In just 0.1 seconds our electronic driver could perform hundreds of calculations like this one (of course depending on how fast the computer is), watching continuously the scenario and possible maneuvers and situations in the near future, evaluating the best possible decision to avoid an accident.

Any numerical integrations have been performed, but the solution of the nonlinear differential equation (19) has been approximated by means of a series expansion.

Similar expressions for any other combination of parameters, excitation or initial conditions could be obtained. These analytic approximated solutions to the nonlinear differential equations, expressed in terms of initial conditions, model parameters and excitation, can be integrated into more complex models of vehicular dynamics.

\section{CONCLUSION}

In this article a methodology has been presented to obtain approximate solutions to some of the equations running the vehicle dynamics through developments in series. These approximate solutions show a very efficient computation and they are adequate for real time calculation in electronic collision avoidance systems. In other words, the solutions of the mechanic behaviour of a car have been adapted to a faster electronic computing.

Some of the approaches of this work to vehicle dynamic simulation are unusual:

- Symbolic computing instead of numeric which is in itself, an innovative approach.

- Obtaining analytic solutions (approximated) even for nonlinear differential equations.

- Pre-calculated solutions.

- Systematic use of polynomial and rational solutions which allows a very fast processing on real time.
- Accurate "enough" solutions, instead of "the most accurate" solutions.

- Solutions valid only within a limited range (of time, of values of the parameters, of frequencies, responses etc), instead of general validity expressions. That means adapted solutions for every situation and/or application.

- "Cascade" symbolic code (see Table IV) with different operations instead of repeated steps of numerical integration. That means a more complicated work of preparation for the engineer, but finally less computational load for the computer.

- Insertion of approximation theory within the process of solving the differential equations.

Using these techniques and methodologies, the following savings of computing time have been obtained in the examples shown:

- $\quad 95 \%$ in the tyre model

- $99.99 \%$ in the steering geometry model

- $\quad 77 \%$ in the vertical model (at least).

- $87 \%$ in the longitudinal model (at least).

Some application examples have been presented.

Although some of the simulation models here presented are not very complex, the main contribution of this article consists of showing the use of theories, techniques and methods of approximation of functions and differential equations in this field, as they have never been applied to vehicle dynamics before.

This fast computing techniques presented here can be applied in the set of strategies and algorithms of the driver-free car, in which the speed of computing is a critical factor, especially if the electronic driver takes the control during active safety and accident avoidance manoeuvers.

As future follow-up for this research, the methodology here proposed can be used in more complex and integrated models, specifically it has to continue integration of the vertical model of half a vehicle (the bicycle model) to lateral dynamics models, to the integration of the tyre model with the two previous ones and, finally, to the integration in the complete model 10 GDL plus the tyre model, using the tools identified in this article.

\section{REFERENCES}

[1] Sivaraman, S. Trivedi. Looking at Vehicles on the Road: A Survey of Vision-Based Vehicle Detection, Tracking, and Behavior Analysis. IEEE T Intell Transp. Volume: 14,No 4, December 2013 1773-1795

[2] Smith, C.E., Richards, C.A., Brandt, S.A., Papanikolopoulos, N.P. Visual tracking for intelligent vehicle-highway systems. IEEE T Veh Technol. Volume: 45, Issue:4. 1996 Pages: 744 - 759. doi: 10.1109/25.543744

[3] Hattori, Y.; Ono,E.; Hosoe,S. "Optimum Vehicle Trajectory Control for Obstacle Avoidance Problem. IEEE/ASME Transactions on Mechatronics. Vol:11, Issue 5; Oct 2006.DOI: 10.1109/TMECH.2006.882981.

[4] Keller, C.G. ; Gavrila, D.M. Will the Pedestrian Cross? A Study on Pedestrian Path Prediction. IEEE T Intell Transp. Volume: PP, Issue: 99 2013. Pages 1-13 doi:10.1109/TITS.2013.2280766

[5] Margolis, L.D. and Blank, M. Minimizing the Path Radius of Curvature for Collision Avoidance. 1. Vehicle Syst. Dyn, Vol 33. pp.183-201 (2000). 
[6] López, A. Predicción de situaciones inseguras en vehículos automóviles. PhD Thesis. Universidad Politécnica de Madrid. (1994).

[7] Moriano, C. Método de procesamiento rápido de las ecuaciones de la dinámica vehicular. PhD Thesis. Universidad Antonio de Nebrija. (2013).

[8] Edwards, C.H. and Penney, D.E. Ecuaciones Diferenciales. Edition Pearson Educación (2001).

[9] Burden, R.L. and Faires, J.D., Numerical Analysis. 6a Edition. J. Ed. Thomson (1998).

[10] Totik, V., 2005. Orthogonal Polynomials. Surveys in Approximation Theory, 1, pp.70-125.

[11] Fox, L. and Parker, I.B., 1968. Chebyshev Polynomials in Numerical Analysis. Oxford Mathematical Handbooks. Oxford University Press (1968).

[12] Handbook of Mathematical functions with Formulas, Graphs and Mathematical Tables, 9th Edition. Dover Publications. New York (1972).

[13] Tawfik, S.A., 2005. Minimax Approximation and Remez Algorithm. http://www.math.unipd.it/ alvise/CS_2008/APPROSSIMAZIONE_200 9/MFILES/Remez.pdf

[14] Higuchi, F., Gofuku, S., Maekawa, Approximation of involute curves for CAD system processing. Engineering With Computers 08/2007; 23(3):207-214. DOI: 10.1007/s00366-007-0060-3

[15] Remez, E.Y., 1934. Sur la détermination des polynômes d'approximation de degré donnée. Comm. Soc. Math. Kharkov, 10, pp.41-63.

[16] Fraser, W., 1965. A survey of methods of computing Minimax and NearMinimax polynomial approximation for functions of a single independent variable. Journal of the ACM, 12 (3), pp.295-314. doi: $10.1145 / 321281.321282$.

[17] Powel, M.J.D.: Approximation Theory and Methods. Cambridge, Cambridge University Press (1981)

[18] Bultheel, A., González Vera, P., Hendriksen, E. and Njastad, O. Orthogonal Rational Functions. Volume 5 of Cambridge Monographs on Applied and Computational Mathematics. Cambridge University Press (1999).

[19] Van Deun, J., Bultheel, A. and González Vera, P., 2005. On computing rational Gauss-Chebyshev quadrature formulas. Math. Comput 01/2006; 75:307-326. doi: 10.1090/S0025-5718-05-01774-6.

[20] Baffet, G. Charara, A. Dherbomez, G. “'An Observer of Tire Road Forces and Friction for Active Security Vehicle Systems", IEEE/ASME Transactions on Mechatronics. Publication Date: Dec. 2007 Volume: 12, Issue: 6 On page(s): 651-661

[21] Doumiati, M. ; Victorino, A.C. ; Charara, A. ; Lechner, D. Onboard RealTime Estimation of Vehicle Lateral Tire-Road Forces and Sideslip Angle. IEEE/ASME Transactions on Mechatronics. Publication Date: 2011 Volume: 16, Issue: 4 On page(s): 601-614

[22] Kanghyun Nam ; Fujimoto, H. ; Hori, Y. Advanced Motion Control of Electric Vehicles Based on Robust Lateral Tire Force Control via Active Front Steering. . IEEE/ASME Transactions on Mechatronics. Publication Year: 2014 Volume: 19, Issue: 1 On page(s): 289-299

[23] Pacejka H.B.: Tire and Vehicle Dynamics. Oxford, Elsevier (2002).

[24] Bakker, E., Nyborg, L., and Pacejka, H.: Tyre Modelling for Use in Vehicle Dynamics Studies, SAE Technical Paper 870421, 1987, doi:10.4271/870421.

[25] Bakker, E., Pacejka, H., and Lidner, L.: A New Tire Model with an Application in Vehicle Dynamics Studies, SAE Technical Paper 890087 , 1989, doi: 10.4271/890087

[26] Vélez, P., Moriano C.: Approximations to the Magic Formula. Int. J Auto. Tech-Kor. Vol 11, n 2 2,155-166, KSAE (2010)

[27] Marín, L., Vallés, M., Soriano, A., Valera, A., Albertos, P. Event-Based Localization in Ackermann Steering Limited Resource Mobile Robots. IEEE/ASME Transactions on Mechatronics. Publication Date: Aug. 2014 Volume: 19, Issue: 4 On page(s): 1171-1182

[28] López, A., Vélez P., Moriano C.: Método de procesamiento rápido de las ecuaciones de la dinámica vehicular mediante polinomios de Chebyshev. Encuentro Internacional de Algebra Computacional. EACA. (2006).

[29] Lanczos, C., 1957. Applied Analysis. Prentice-Hall New York, Pitman London.
[30] López, A., Moriano C.: Simulation of the suspension of a vehicle using Chebyshev series. P I Mech. Eng. D-J Auto., March 2014, Vol.228 doi: $10.1177 / 0954407013513569$.

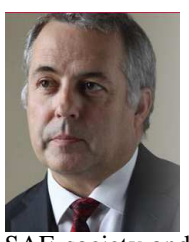

Alberto López

First author. Received his Industrial Engineering (Mechanics Intensification) and the PhD. Degrees from Universidad Politécnica de Madrid- Spain.

$\mathrm{He}$ is professor of Mechanism and Machine Theory at the Nebrija University. His PhD. Work (1995) was devoted to collision avoidance systems. He is an active member of the SAE society and president of the education branch of the ASEPA (Spanish Association of Automotive Professionals).

Alberto is Director of the Polytechnic School of Engineering of the Nebrija University and its PhD Program.

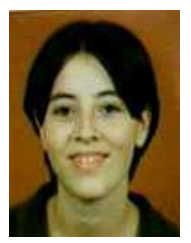

Cristina Moriano

Received her Industrial Engineering and PhD. Degrees from the Universidad Antonio de Nebrija in Madrid-Spain.

She is a professor of Mechanism and Machine Theory at the Nebrija University. Her PhD. Work (2013) was focused on fast computing methods for vehicle dynamics. Cristina is currently working on advanced active safety within the research group of Automotive Engineering.

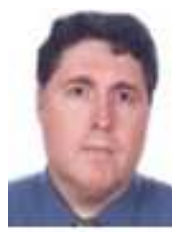

José Luis Olazagoitia

Received his Industrial Engineering (Mechanics Intensification) and the PhD. Degrees from the Universidad de Navarra in San Sebastian - Spain. He is professor of "Machine Calculation, Design and Testing" at the Nebrija University. His PhD. Work (1999) was focused on Optimization Techniques applied to the design of mechanisms.

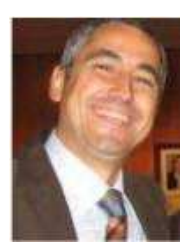

Francisco Javier Páez

$\mathrm{He}$ is $\mathrm{PhD}$ Mechanical Engineer and an Associate Professor of Universidad Politécnica de Madrid in Spain. He is Deputy Director of Quality, Training and Dissemination at the University Institute for Automobile Research at Technical University of Madrid (INSIA-UPM). He has over 17 years of experience in road accident research including in-depth accident investigation studies. Javier is the Spanish Member of the European Enhanced Vehicle-safety Committee -Working Group 21 (Accident Studies), and he has participated in several European Research Projects of the IV, V and VII Framework Program (European Commission), and of the National Competitive Programs. 